American Journal of Applied Sciences 3 (12): 2184-2189, 2006

ISSN 1546-9239

(C) 2006 Science Publications

\title{
Resonance in Satellite's Motion Under Air Drag
}

\author{
Rashmi Bhardwaj and Mani Sethi \\ School of Basic and Applied Sciences Department of Mathematics \\ Guru Gobind Singh Indraprastha University, Kashmere Gate, Delhi, India
}

\begin{abstract}
This article studies the attitude motion of a satellite in a circular orbit under the influence of central body of mass $\mathrm{M}$ and its moon of mass $\mathrm{m}$, whose orbit is assumed to be circular and coplanar with the orbit of the satellite. The body is assumed to be tri-axial body with principal moments of inertia $\mathrm{A}<\mathrm{B}<\mathrm{C}$ at its centre of mass, $\mathrm{C}$ is the moment of inertia about the spin axis which is perpendicular to the orbital plane. These principal axes are taken as the co-ordinate axes $\mathrm{x}, \mathrm{y}, \mathrm{z}$; the $\mathrm{z}$ axis being perpendicular to the orbital plane. We have studied the rotational motion of satellite in the circular orbit under the influence of aerodynamic torque. Using BKM method, it is observed that the amplitude of the oscillation remains constant upto the second order of approximation. The main and the parametric resonance have been shown to exist and have been studied by BKM method. The analysis regarding the stability of the stationary planar oscillation of a satellite near the resonance frequency shows that the discontinuity occurs in the amplitude of the oscillation at a frequency of the external periodic force which is less than the frequency of the natural oscillation.
\end{abstract}

Key words: Aerodynamic torque, non -linear, planar oscillation, resonance

\section{INTRODUCTION}

The determination and prediction of the orbit of a satellite in the near-earth environment is complicated by the fact that the satellite is influenced by the dissipative effects of the earth's atmosphere. For many artificial satellites, this fluctuation in the drag is a fundamental source of error in the orbital predictions. The study of dynamics of rotating bodies has been studied by Inarrea and Lanchares ${ }^{[1]}$ under the influence of aerodynamic drag. Abd. et al. ${ }^{[2]}$ have constructed a second order atmospheric drag theory based on the usage of TD 88 model. Maciejewski \& Przybylska ${ }^{[3]}$ analyzed the integrability of a dynamical system under the influence of gravitational and magnetic fields. Barkin, Ferrandiz ${ }^{[4]}$ discussed Resonant Rotation of Two-layer Moon and Mercury. Callegari, FerrazMello, Michtchenko ${ }^{[5]}$, discussed the Dynamics of Two Planets in the 3/2 Mean-motion Resonance in Application to the Planetary System of the Pulsar PSR $\mathrm{B} 1257+12$. Beaugé et al. ${ }^{[6,7]}$ studied Planetary migration and extra solar planets in the $2 / 1$ meanmotion resonance. They reviewed recent results on the dynamics of multiple-planet extra-solar systems, including main sequence stars and the pulsar PSR B1257+12 and comparatively, our own Solar System. They discussed Resonances and stability of extra-solar planetary systems. Massimiliano ${ }^{[8,9]}$, numerically detected the web of three-planet resonances (i.e., resonances among mean anomalies, nodes and perihelia of three planets) with respect to the variation of the semi-major axis of Saturn and Jupiter, in a model including the planets from Jupiter to Neptune. Zhou et $a l .{ }^{[10,11]}$ showed that the occurrence of apsidal secular resonance depends only on the mass ratio semi-major rate and eccentricity rate between the two planets. Yokoyama et al. ${ }^{[12]}$ have shown that once captured in the resonance, the inclination of the satellite reaches very high values. But none of them have studied the effect of aerodynamic torque on the attitude motion of a satellite in circular orbit. Using BKM method, we have discussed that the amplitude of the oscillation remains constant upto the second order of approximation.

Equation of motion: Let $\overline{\mathrm{r}}$ be the instantaneous radius vector of the centre of mass of the satellite, $\theta$ be the angle that the long axis of the satellite makes with a fixed line EF lying in the orbital plane and $\delta / 2$ the angle between the radius vector $\bar{r}$ and the long axis.

Euler's equation of motion about z-axis, taking $v$ (true anomaly as an independent variable) is obtained as:

$\frac{d^{2} \eta}{d v^{2}}+\omega^{2} \sin \eta-\varepsilon\left(v^{2}-b v-d\right) \sin v=0$

where $\omega^{2}=\frac{3(B-A)}{C}, \varepsilon=\frac{\rho \mathrm{SC}_{\mathrm{d}} \mathrm{a}^{2}}{\mathrm{C}} \times \frac{1}{\Omega^{4}}$

$$
\mathrm{b}=\frac{2 \Omega \mathrm{V}_{1}-\Omega 1 \omega}{\mathrm{a}} \text { and } \mathrm{d}=\frac{\mathrm{V}_{1}{ }^{2} \Omega^{2}-\Omega^{2} \mathrm{~V}_{1} 1 \omega}{\mathrm{a}^{2}} \text { are all }
$$

constants

Non -resonant planar oscillation of a satellite: In the equation (1), the non linearity $[\eta-\sin \eta]$ is taken 
sufficiently weak and therefore it can also be taken of the order of $\varepsilon$. Therefore by taking $\omega^{2}=c \varepsilon$ and using BKM method to solve

$\frac{\mathrm{d}^{2} \eta}{\mathrm{dv}^{2}}+\omega^{2} \eta=\varepsilon f(\mathrm{v}, \eta)$

where $f(v, \eta)=c[\eta-\sin \eta]+\left(v^{2}-b v-d\right) \sin v$, the solution is obtained as

$\eta=\mathrm{a} \cos \psi+\varepsilon \mathrm{u}_{1}(\mathrm{a}, \mathrm{v}, \psi)+\varepsilon^{2} \mathrm{u}_{2}(\mathrm{a}, \mathrm{v}, \psi)+$

where the amplitude $a$ and the phase $\psi$ are determined by differential equations

$$
\begin{aligned}
& \frac{d a}{d v}=\varepsilon A_{1}(a)+\varepsilon^{2} A_{2}(a)+ \\
& \frac{d \psi}{d v}=\omega+\varepsilon B_{1}(a)+\varepsilon^{2} B_{2}(a)+
\end{aligned}
$$

In our problem

$$
\begin{aligned}
& \mathrm{A}_{1}(\mathrm{a})=0 \\
& \mathrm{~B}_{1}(\mathrm{a})=\frac{\mathrm{c}}{2 \mathrm{a} \omega}\left[2 \mathrm{~J}_{1}(\mathrm{a})-\mathrm{a}\right] \\
& \mathrm{u}_{1}(\mathrm{a}, \mathrm{v}, \psi)=\frac{1}{\omega^{2}-1}\left[\mathrm{v}^{2} \sin \mathrm{v}-\frac{2\left(\omega^{2}+1\right)}{\left(\omega^{2}-1\right)^{2}} \sin \mathrm{v}-\frac{4 \mathrm{v} \cos \mathrm{v}}{\omega^{2}-1}\right] \\
& -\frac{b}{\omega^{2}-1}\left[v \sin v-\frac{2 \cos v}{\omega^{2}-1}\right] \\
& -\frac{\mathrm{d} \sin \mathrm{v}}{\omega^{2}-1}+\frac{\mathrm{c}}{2 \omega^{2}} \sum_{\mathrm{k}=1}^{\infty}(-1)^{\mathrm{ki}} \mathrm{J}_{2 \mathrm{k}+1}(\mathrm{a}) \frac{\cos (2 \mathrm{k}+1) \psi}{\mathrm{k}(\mathrm{k}+1)} \\
& \mathrm{A}_{2}(\mathrm{a})=0 \\
& B_{2}(a)=\frac{c^{2}}{2 a \omega^{3}} \sum_{k=1}^{\infty} \frac{J_{2 k+1}(a) J_{2 k+1}^{\prime}(a)}{k(k+1)}-\frac{c^{2}}{8 a^{2} \omega^{3}}\left[2 J_{1}(a)-a\right]^{2} \\
& \text { Bessel's function of order } k \text {. } \\
& \mathrm{u}_{2}(\mathrm{a}, \mathrm{v}, \psi)=\frac{-\mathrm{c}^{2}}{8 \mathrm{a} \omega^{4}}\left[2 \mathrm{~J}_{1}(\mathrm{a})-\mathrm{a}\right] \\
& \sum_{\mathrm{k}=1}^{\infty}(-1)^{\mathrm{k}}(2 \mathrm{k}+1)^{2} \mathrm{~J}_{2 \mathrm{k}+1}(\mathrm{a}) \frac{\cos (2 \mathrm{k}+1) \psi}{\mathrm{k}^{2}(\mathrm{k}+1)^{2}} \\
& -\frac{\mathrm{c}^{2}}{8 \omega^{4}} \sum_{\mathrm{k}=1}^{\infty}(-1)^{\mathrm{k}} \mathrm{J}_{2 \mathrm{k}+1}(\mathrm{a}) \frac{\cos (2 \mathrm{k}+1) \psi}{\mathrm{k}^{2}(\mathrm{k}+1)^{2}} \\
& +\frac{c v^{2} \sin v}{\left(\omega^{2}-1\right)^{2}}\left(1-J_{0}(a)\right) \\
& -\frac{b c v \sin v\left(1-\mathrm{J}_{0}(\mathrm{a})\right)}{\left(\omega^{2}-1\right)^{2}} \\
& -\frac{c d \sin v\left(1-J_{0}(a)\right)}{\left(\omega^{2}-1\right)^{2}}-\frac{8 c v \cos v}{\left(\omega^{2}-1\right)^{3}}\left(1-J_{0}(a)\right) \\
& -\frac{4 \mathrm{c}\left(\omega^{2}+3\right) \sin \mathrm{v}}{\left(\omega^{2}-1\right)^{4}}\left(1-\mathrm{J}_{0}(\mathrm{a})\right)
\end{aligned}
$$$$
\mathrm{J}_{2 \mathrm{k}+1}^{\prime}(\mathrm{a})=\frac{1}{2}\left[\mathrm{~J}_{2 \mathrm{k}}(\mathrm{a})-\mathrm{J}_{2 \mathrm{k}+2}(\mathrm{a})\right] \quad \text { where } \quad \mathrm{J}_{\mathrm{k}}(\mathrm{a}) \quad \text { stands }
$$

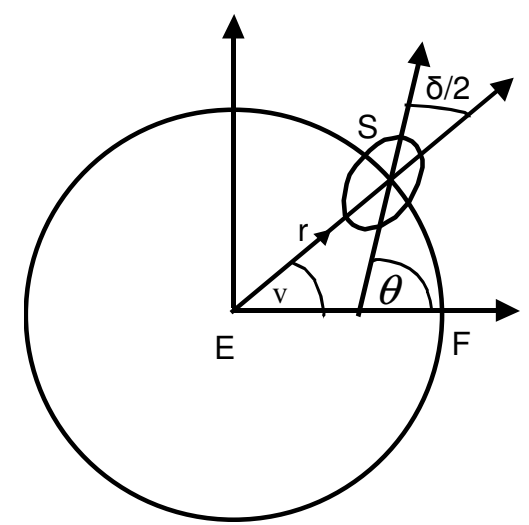

Fig. 1: Satellite planar oscillation in circular orbit

$$
\begin{aligned}
& -\frac{\mathrm{c}}{\omega^{2}-1} \sum_{\mathrm{k}=1}^{\infty}(-1)^{\mathrm{k}} \mathrm{J}_{2 \mathrm{k}}(\mathrm{a})\left[\frac{\mathrm{v}^{2} \sin (\mathrm{v}+2 \mathrm{k} \psi)}{(\omega(2 \mathrm{k}+1)+1)(\omega(1-2 \mathrm{k})-1)}\right. \\
& +\frac{\mathrm{v}^{2} \sin (\mathrm{v}-2 \mathrm{k} \psi)}{(\omega(2 \mathrm{k}+1)-1)(\omega(1-2 \mathrm{k})+1)} \\
& -\frac{2 \sin (\mathrm{v}+2 \mathrm{k} \psi)+4(1+2 \mathrm{k} \omega) \mathrm{v} \cos (\mathrm{v}+2 \mathrm{k} \psi)}{(\omega(2 \mathrm{k}+1)+1)^{2}(\omega(1-2 \mathrm{k})-1)^{2}} \\
& -\frac{4(1+2 \mathrm{k} \omega)^{2} \sin (\mathrm{v}+2 \mathrm{k} \psi)}{(\omega(2 \mathrm{k}+1)+1)^{3}(\omega(1-2 \mathrm{k})-1)^{3}} \\
& -\frac{2 \sin (\mathrm{v}-2 \mathrm{k} \psi)+4(1-2 \mathrm{k} \omega) \mathrm{v} \cos (\mathrm{v}-2 \mathrm{k} \psi)}{(\omega(2 \mathrm{k}+1)-1)^{2}(\omega(1-2 \mathrm{k})+1)^{2}} \\
& \left.-\frac{4(1-2 \mathrm{k} \omega)^{2} \sin (\mathrm{v}-2 \mathrm{k} \psi)}{(\omega(2 \mathrm{k}+1)-1)^{3}(\omega(1-2 \mathrm{k})+1)^{3}}\right] \\
& +\frac{2 \mathrm{c}\left(\omega^{2}+1\right)}{\left(\omega^{2}-1\right)^{3}} \sum_{\mathrm{k}=1}^{\infty}(-1)^{\mathrm{k}} \mathrm{J}_{2 \mathrm{k}}(\mathrm{a}) \\
& \left.+\frac{\sin (\mathrm{v}+2 \mathrm{k} \psi)}{(\omega(2 \mathrm{k}+1)-1)^{2}(\omega(1-2 \mathrm{k})+1)^{2}}\right] \\
& +\frac{2(1+2 \mathrm{k} \omega) \sin (\mathrm{v}+2 \mathrm{k} \psi)}{(\omega(2 \mathrm{k}+1)+1)(\omega(1-2 \mathrm{k})-1)} \\
& +\frac{4 \mathrm{c}}{\left(\omega \omega^{2}-1\right)^{2} \sum_{\mathrm{k}=1}^{\infty}(-1)^{\mathrm{k}} \mathrm{J}_{2 \mathrm{k}}(\mathrm{a})} \\
& +\frac{\mathrm{v}(\cos (\mathrm{v}+2 \mathrm{k} \psi)}{(\omega(2 \mathrm{k}+1)+1)(\omega(1-2 \mathrm{k}-2 \mathrm{k} \psi)} \\
& +\frac{\mathrm{v} \cos (\mathrm{v}-2 \mathrm{k} \psi)}{(\omega \mathrm{k}+1)-1)(\omega(1-2 \mathrm{k})+1)} \\
& +\frac{2 \mathrm{k})+1)(\omega(1-2 \mathrm{k})+1)}{(\omega)}
\end{aligned}
$$




$$
\begin{aligned}
& +\frac{\mathrm{bc}}{\omega^{2}-1} \sum_{\mathrm{k}=1}^{\infty}(-1)^{\mathrm{k}} \mathrm{J}_{2 \mathrm{k}}(\mathrm{a}) \\
& {\left[\frac{\mathrm{v} \sin (\mathrm{v}+2 \mathrm{k} \psi)}{(\omega(2 \mathrm{k}+1)+1)(\omega(1-2 \mathrm{k})-1)}\right.} \\
& +\frac{2(1+2 \mathrm{k} \omega) \cos (\mathrm{v}+2 \mathrm{k} \psi)}{(\omega(2 \mathrm{k}+1)+1)^{2}(\omega(1-2 \mathrm{k})-1)^{2}} \\
& +\frac{\mathrm{v} \sin (\mathrm{v}-2 \mathrm{k} \psi)}{(\omega(2 \mathrm{k}+1)-1)(\omega(1-2 \mathrm{k})+1)}
\end{aligned}
$$$$
\left.+\frac{2(1-2 \mathrm{k} \omega) \cos (\mathrm{v}-2 \mathrm{k} \psi)}{(\omega(2 \mathrm{k}+1)-1)^{2}(\omega(1-2 \mathrm{k})+1)^{2}}\right]
$$$$
+\frac{2 \mathrm{bc}}{\left(\omega^{2}-1\right)^{2}} \sum_{\mathrm{k}=1}^{\infty}(-1)^{\mathrm{k}} \mathrm{J}_{2 \mathrm{k}}(\mathrm{a})
$$$$
\left[\frac{\cos (\mathrm{v}+2 \mathrm{k} \psi)}{(\omega(2 \mathrm{k}+1)+1)(\omega(1-2 \mathrm{k})-1)}\right.
$$$$
\left.+\frac{\cos (\mathrm{v}-2 \mathrm{k} \psi)}{(\omega(2 \mathrm{k}+1)-1)(\omega(1-2 \mathrm{k})+1)}\right]
$$$$
+\frac{\mathrm{cd}}{\omega^{2}-1} \sum_{\mathrm{k}=1}^{\infty}(-1)^{\mathrm{k}} \mathrm{J}_{2 \mathrm{k}}(\mathrm{a})
$$$$
\left[\frac{\sin (\mathrm{v}+2 \mathrm{k} \psi)}{(\omega(2 \mathrm{k}+1)+1)(\omega(1-2 \mathrm{k})-1)}\right.
$$$$
\left.+\frac{\sin (\mathrm{v}-2 \mathrm{k} \psi)}{(\omega(2 \mathrm{k}+1)-1)(\omega(1-2 \mathrm{k})+1)}\right]
$$$$
+\frac{c^{2} J_{0}(a)}{8 \omega^{4}} \sum_{k=1}^{\infty}(-1)^{k} \frac{J_{2 k+1}(a) \cos (2 k+1) \psi}{k^{2}(k+1)^{2}}
$$$$
+\frac{c^{2}}{8 \omega^{4}} \sum_{\substack{l, k=1 \\ l \neq k, k+1}}^{\infty} \frac{(-1)^{l+k} J_{2 k+1}(a) J_{2 l}(a)}{k(k+1)}
$$$$
\left[\frac{\cos (2 k+2 l+1) \psi}{(l+k)(l+k+1)}+\frac{\cos (2 k-2 l+1) \psi}{(k-l)(k+1-l)}\right]
$$

Thus in the first approximation, the solution is given by

$$
\eta=a \cos \psi
$$

where the amplitude $a$ and the phase $\psi$ are given by

$$
\begin{aligned}
& \frac{\mathrm{da}}{\mathrm{dv}}=0 \Rightarrow \mathrm{a}=\text { constant }, \\
& \frac{\mathrm{d} \psi}{\mathrm{dv}}=\omega+\frac{\omega}{2 \mathrm{a}}\left[2 \mathrm{~J}_{1}(\mathrm{a})-\mathrm{a}\right]
\end{aligned}
$$

and in the second approximation, the solution is obtained as

$$
\begin{aligned}
& \eta=a \cos \psi+\frac{\varepsilon}{\omega^{2}-1} \\
& {\left[v^{2} \sin v-\frac{2\left(\omega^{2}+1\right)}{\left(\omega^{2}-1\right)^{2}} \sin v-\frac{4 v \cos v}{\omega^{2}-1}\right]} \\
& -\frac{b \varepsilon}{\omega^{2}-1}\left[v \sin v-\frac{2 \cos v}{\omega^{2}-1}\right] \\
& -\frac{d \varepsilon \sin v}{\omega^{2}-1}+\frac{\varepsilon c}{2 \omega^{2}} \sum_{k=1}^{\infty}(-1)^{k i} \\
& J_{2 k+1}(a) \frac{\cos (2 k+1) \psi}{k(k+1)}
\end{aligned}
$$

where the amplitude $a$ and the phase $\psi$ are given by

$$
\begin{aligned}
& \frac{\mathrm{da}}{\mathrm{dv}}=0 \Rightarrow \mathrm{a}=\text { constant } \\
& \frac{\mathrm{d} \psi}{\mathrm{dv}}=\omega+\frac{\omega}{2 \mathrm{a}}\left[2 \mathrm{~J}_{1}(\mathrm{a})-\mathrm{a}\right] \\
& +\frac{\omega}{2 a} \sum_{k=1}^{\infty} \frac{J_{2 k+1}(a) J_{2 k+1}^{\prime}(a)}{k(k+1)}-\frac{\omega}{8 a^{2}}\left[2 J_{1}(a)-a\right]^{2}
\end{aligned}
$$

From the equations (8) \& (10), we observe that the amplitude of the oscillation remains constant even upto the second order of approximation of the aerodynamic torque parameter $\varepsilon$ and the equation (7) gives us the main resonance at $\omega= \pm 1$ and the parametric resonance at $\omega= \pm \frac{1}{2 \mathrm{k}+1}, \mathrm{k} \in \mathrm{I}$.

Resonant planar oscillation of a satellite: We proceed to construct the asymptotic solution of the system in the general case, which is valid at and near the resonance $\omega \cong k$ exploiting the well known BKM method.

For $\mathcal{E}=0$ the generating solution is given by

$\eta=a \cos \psi, \psi=k v+\theta$

where the amplitude $a$ and the phase $\theta$ are determined by differential equations

$$
\begin{aligned}
& \frac{\mathrm{da}}{\mathrm{dv}}=\varepsilon \mathrm{A}_{1}(\mathrm{a}, \theta) \\
& \frac{\mathrm{d} \theta}{\mathrm{dv}}=\omega-\mathrm{k}+\varepsilon \mathrm{B}_{1}(\mathrm{a}, \theta) \\
& \frac{d \psi}{d v}=\omega+\varepsilon B_{1}(a, \theta)
\end{aligned}
$$

where $\mathrm{A}_{1}(\mathrm{a}, \theta)$ and $\mathrm{B}_{1}(\mathrm{a}, \theta)$ are periodic w.r.t. $\theta$. Using (11) and (12), we calculate $\frac{\mathrm{d} \eta}{\mathrm{dv}}$ and $\frac{\mathrm{d}^{2} \eta}{\mathrm{dv}^{2}}$ and then substituting the values of $\eta, \frac{d \eta}{d v}$ and $\frac{d^{2} \eta}{d v^{2}}$ 
in the equation (12) and equating the coefficients of $\varepsilon$, we get

$\left(\omega \frac{\partial \mathrm{A}_{1}}{\partial \theta}-\mathrm{k} \frac{\partial \mathrm{A}_{1}}{\partial \theta}-2 \mathrm{a} \omega \mathrm{B}_{1}\right) \cos \psi-\left(2 \omega \mathrm{A}_{1}+\mathrm{a} \omega \frac{\partial \mathrm{B}_{1}}{\partial \theta}-\mathrm{ak} \frac{\partial \mathrm{B}_{1}}{\partial \theta}\right) \sin \psi$

$=c[a \cos \psi-\sin (a \cos \psi)]+\left(v^{2}-b v-d\right) \sin v$

Using Fourier expansion given by

$\sin [\mathrm{a} \cos \psi]=2 \sum_{\mathrm{k}=0}^{\infty}(-1)^{\mathrm{k}} \mathrm{J}_{2 \mathrm{k}+1}(\mathrm{a}) \cos (2 \mathrm{k}+1) \psi$

$\cos [\mathrm{a} \cos \psi]=\mathrm{J}_{0}(\mathrm{a})+2 \sum_{\mathrm{k}=1}^{\infty}(-1)^{\mathrm{k}} \mathrm{J}_{2 \mathrm{k}}(\mathrm{a}) \cos (2 \mathrm{k}) \psi$

where $\mathbf{J}_{\mathrm{k}}$ (a) stands Bessel's function of order $\mathrm{k}$, In the equation (13) and then comparing the coefficients of $\cos \psi$ and $\sin \psi$, we get

$$
\begin{aligned}
& (\omega-\mathrm{k}) \frac{\partial \mathrm{A}_{1}}{\partial \theta}-2 \mathrm{a} \omega \mathrm{B}_{1}=\mathrm{c}\left[\mathrm{a}-2 \mathrm{~J}(\mathrm{a})_{1}\right]-\theta^{2} \sin \theta-\mathrm{b} \theta \sin \theta+\mathrm{d} \sin \theta \\
& a(\omega-k) \frac{\partial B_{1}}{\partial \theta}+2 \omega A_{1}=-\theta^{2} \cos \theta-b \theta \cos \theta+d \cos \theta(14)
\end{aligned}
$$

Solving the equation (14) taking $\mathrm{k}=1$, we get

$\mathrm{A}_{1}=\frac{-1}{\omega+1}\left[\theta^{2} \cos \theta-\frac{2(\omega-1)^{2} \cos \theta}{(3 \omega-1)(\omega+1)}-\frac{4(\omega-1)^{4} \cos \theta}{(3 \omega-1)^{2}(\omega+1)^{2}}+\frac{4(\omega-1)^{2} \theta \sin \theta}{(3 \omega-1)(\omega+1)}\right]$

$$
-\frac{2(\omega-1)}{(3 \omega-1)(\omega+1)}\left[\theta \sin \theta-\frac{2(\omega-1)^{2} \cos \theta}{(3 \omega-1)(\omega+1)}\right]
$$

$-\frac{b}{\omega+1}\left[\theta \cos \theta+\frac{2(\omega-1)^{2} \sin \theta}{(3 \omega-1)(\omega+1)}\right]+\frac{d \cos \theta}{\omega+1}-\frac{b(\omega-1) \sin \theta}{(3 \omega-1)(\omega+1)}$

$\mathrm{B}_{1}=\frac{1}{\mathrm{a}(\omega+1)}\left[\theta^{2} \sin \theta-\frac{2(\omega-1)^{2} \sin \theta}{(3 \omega-1)(\omega+1)}-\frac{4(\omega-1)^{4} \sin \theta}{(3 \omega-1)^{2}(\omega+1)^{2}}-\frac{4(\omega-1)^{2} \theta \cos \theta}{(3 \omega-1)(\omega+1)}\right]$

$-\frac{2(\omega-1)}{\mathrm{a}(3 \omega-1)(\omega+1)}\left[\theta \cos \theta+\frac{2(\omega-1)^{2} \sin \theta}{(3 \omega-1)(\omega+1)}\right]$

$+\frac{b}{a(\omega+1)}\left[\theta \sin \theta-\frac{2(\omega-1)^{2} \cos \theta}{(3 \omega-1)(\omega+1)}\right]$

$-\frac{b(\omega-1) \cos \theta}{a(3 \omega-1)(\omega+1)}-\frac{d \sin \theta}{a(\omega+1)}-\frac{c}{2 a \omega}\left[a-2 J_{1}(a)\right]$

Thus the solution in the first approximation is given by $\eta=\mathrm{a} \cos (\mathrm{v}+\theta)$

where the amplitude ' $a$ ' and the phase' $\theta$ ' are the solutions of the system

$$
\begin{aligned}
& \frac{\mathrm{da}}{\mathrm{dv}}=\frac{-\varepsilon}{\omega+1}\left[\theta^{2} \cos \theta-\frac{2(\omega-1)^{2} \cos \theta}{(3 \omega-1)(\omega+1)}-\frac{4(\omega-1)^{4} \cos \theta}{(3 \omega-1)^{2}(\omega+1)^{2}}+\frac{4(\omega-1)^{2} \theta \sin \theta}{(3 \omega-1)(\omega+1)}\right] \\
& -\frac{2 \varepsilon(\omega-1)}{(3 \omega-1)(\omega+1)}\left[\theta \sin \theta-\frac{2(\omega-1)^{2} \cos \theta}{(3 \omega-1)(\omega+1)}\right] \\
& -\frac{\mathrm{b} \varepsilon}{\omega+1}\left[\theta \cos \theta+\frac{2(\omega-1)^{2} \sin \theta}{(3 \omega-1)(\omega+1)}\right]+\frac{\mathrm{d} \varepsilon \cos \theta}{\omega+1}-\frac{\mathrm{b} \varepsilon(\omega-1) \sin \theta}{(3 \omega-1)(\omega+1)} \\
& \frac{\mathrm{d} \theta}{\mathrm{dv}}=(\omega-1)+\frac{\varepsilon}{\mathrm{a}(\omega+1)}\left[\begin{array}{l}
\theta^{2} \sin \theta-\frac{2(\omega-1)^{2} \sin \theta}{(3 \omega-1)(\omega+1)}- \\
-\frac{4(\omega-1)^{4} \sin \theta}{(3 \omega-1)^{2}(\omega+1)^{2}}-\frac{4(\omega-1)^{2} \theta \cos \theta}{(3 \omega-1)(\omega+1)}
\end{array}\right]
\end{aligned}
$$

$$
\begin{aligned}
& -\frac{2 \varepsilon(\omega-1)}{\mathrm{a}(3 \omega-1)(\omega+1)}\left[\theta \cos \theta+\frac{2(\omega-1)^{2} \sin \theta}{(3 \omega-1)(\omega+1)}\right] \\
& +\frac{\mathrm{b} \varepsilon}{\mathrm{a}(\omega+1)}\left[\theta \sin \theta-\frac{2(\omega-1)^{2} \cos \theta}{(3 \omega-1)(\omega+1)}\right] \\
& -\frac{b \varepsilon(\omega-1) \cos \theta}{a(3 \omega-1)(\omega+1)}-\frac{d \varepsilon \sin \theta}{a(\omega+1)}-\frac{\omega}{2 a}\left[a-2 J_{1}(a)\right]
\end{aligned}
$$

The equations (16) cannot be integrated in a closed form due to the dependence of the right hand side on ' $a$ ' and ' $\theta$ '. The stationary state of the oscillation is defined by

$\frac{\mathrm{da}}{\mathrm{dv}}=0=\frac{\mathrm{d} \theta}{\mathrm{dv}}$

However equation (16), may be represented, correct to the second order in the form:

$$
\begin{aligned}
& 2 \frac{d a}{d v}=-\varepsilon\left(\theta^{2}+b \theta+d\right) \cos \theta \\
& 2 a \frac{d \theta}{d v}=a\left(\omega_{e}^{2}-1\right)+\varepsilon \sin \theta\left(\theta^{2}+b \theta-d\right)
\end{aligned}
$$

where $\omega_{e}(a)=\omega-\frac{\omega}{2 a}\left[a-2 J_{1}(a)\right]$

Now, the stationary regimes are given by $\left(\theta^{2}+b \theta+d\right) \cos \theta=0 \Rightarrow \cos \theta=0$,

$\mathrm{a}\left(\omega_{\mathrm{e}}{ }^{2}-1\right)+\varepsilon \sin \theta\left(\theta^{2}+\mathrm{b} \theta-\mathrm{d}\right)=0$

Eliminating phase $\theta$, we get

$\omega_{\mathrm{e}}^{2}-1=-\frac{\varepsilon}{\mathrm{a}}$

Putting $1=\omega+\delta,(\delta<<1)$, we obtain

$\delta^{2}+2 \omega \delta+\frac{\omega^{2} \mathrm{a}^{2}}{8}-\frac{\varepsilon}{\mathrm{a}}=0$,

$\Rightarrow \delta=-\omega \pm \frac{1}{2 \sqrt{2}}\left[8 \frac{\varepsilon}{a}-\omega^{2}\left(a^{2}-8\right)\right]^{1 / 2}$

Let us, now examine the relation existing between the parameters of the system for the occurrence of the effect under consideration. The necessary condition for instability (Jump and Fall) is $\frac{\mathrm{d} \delta}{\mathrm{da}}=0$. Proceeding with equation (20), we get

$$
8 \delta^{2}+16 \omega \delta+3 \omega^{2} a^{2}=0
$$

Since values of $\delta$ are both negative, so that the effect occurs only at a frequency of external periodic force which is less than the frequency of the natural oscillation of the system. Now, maximum value of the amplitude is obtained by the condition $\frac{\mathrm{da}}{\mathrm{d} \delta}=0$, which gives $16 \mathrm{a}(\omega+\delta)=0$, since $\mathrm{a}>0 \Rightarrow \delta=-\omega$. Substituting this value in equation (21) and putting $\omega=1$, the maximum value of a is obtained for different values of $\varepsilon$ from equation 

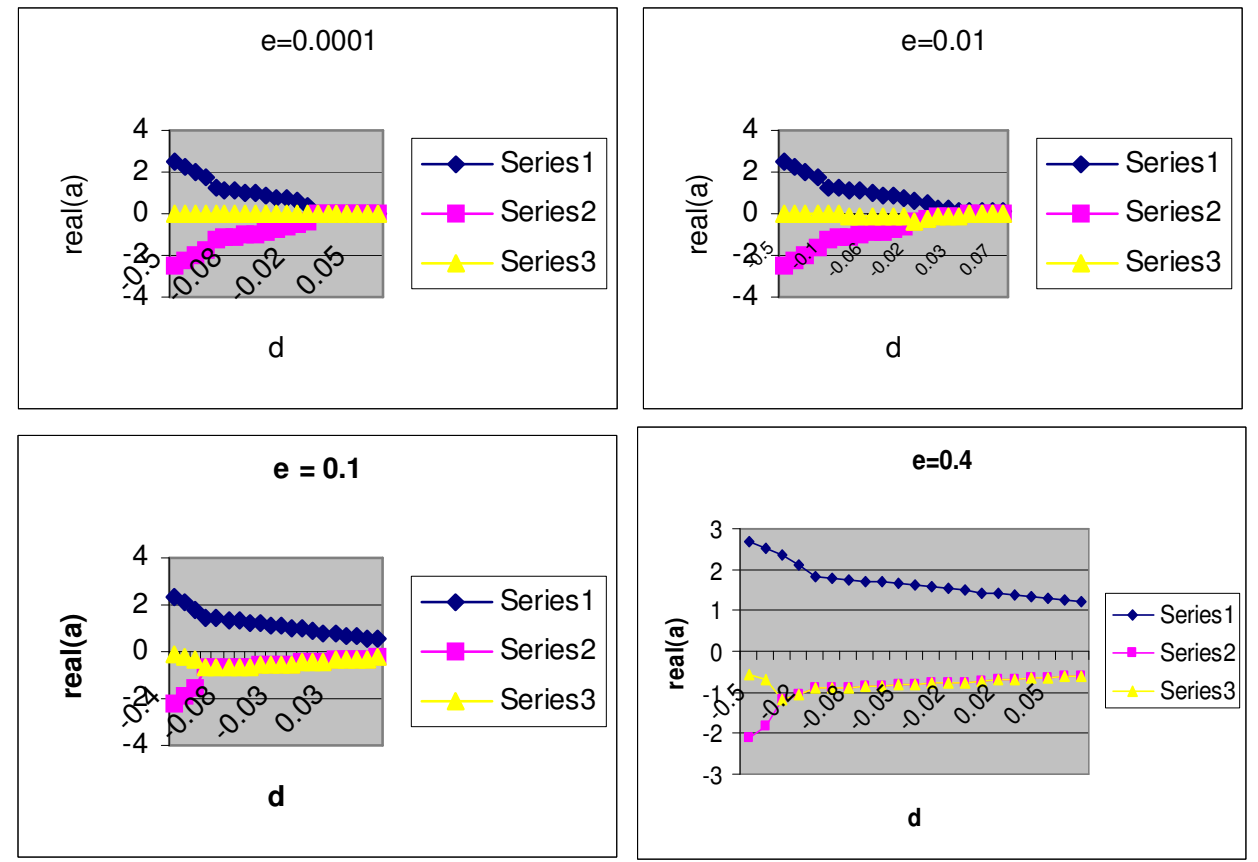

Fig. 2: Resonance curves in case of main resonance for e=0.0001, $0.010 .1,0.4$ at $\omega=1$
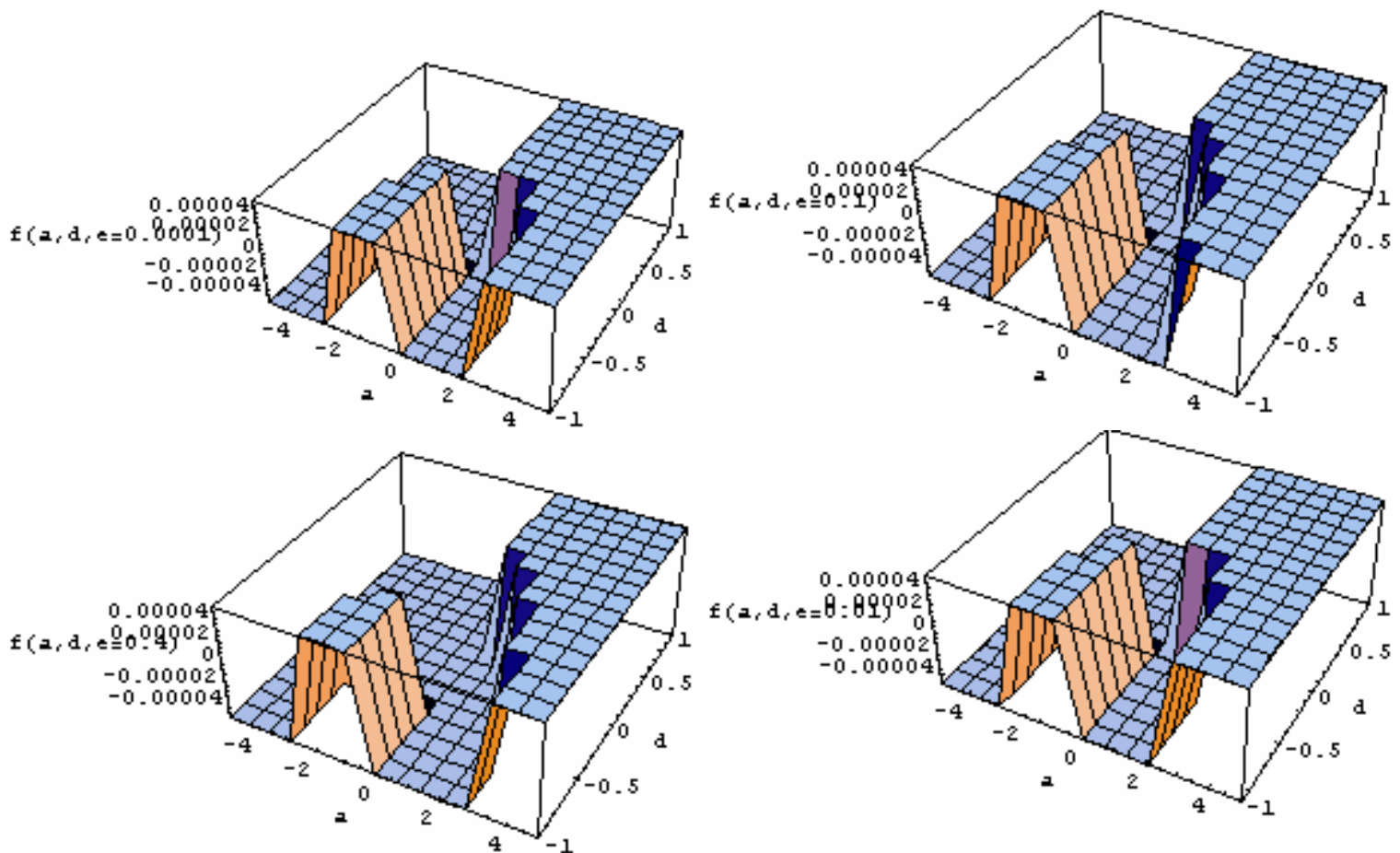

Fig. 3: 3D plot of Resonance curve for $\mathrm{e}=0.0001,0.01,0.1,0.4$ at $\omega=1$

Table 1: Critical values of A

\begin{tabular}{lclll}
\hline $\mathrm{e}$ & $\mathrm{d}$ & $\mathrm{Ad}$ & Ad' $^{\prime}$ & Jump \\
\hline 0.0000001 & 0.01 & $4.98 \mathrm{E}-06$ & $-2.49 \mathrm{E}-06$ & $7.46 \mathrm{E}-06$ \\
0.0001 & 0.01 & $4.97 \mathrm{E}-03$ & $-2.49 \mathrm{E}-03$ & $7.46 \mathrm{E}-03$ \\
0.01 & -0.02 & 0.661603 & -0.3308 & $9.92 \mathrm{E}-01$ \\
0.1 & -0.1 & 1.440599 & -0.7203 & $2.16 \mathrm{E}+00$ \\
0.4 & -0.3 & 2.334662 & -1.16733 & $3.50 \mathrm{E}+00$ \\
0.7 & -0.4 & 2.68442 & -1.34221 & $4.03 \mathrm{E}+00$ \\
0.9 & -0.5 & 2.868936 & -1.43447 & $4.30 \mathrm{E}+00$ \\
\hline
\end{tabular}

$a^{3}-8 a-8 \varepsilon=0$, which is tabulated in Table 1 and the jump was shown with $2 \mathrm{D}$ and $3 \mathrm{D}$ resonance curves.

\section{CONCLUSION}

The attitude motion of a satellite in circular orbit under aerodynamic torque is obtained. Using BKM 
method, we have observed that the amplitude of the oscillation remains constant upto the second order of approximation. The main resonance occurs at $\omega= \pm 1$ and the parametric resonance at

$\omega= \pm \frac{1}{2 k+1}, k \in I$

At main resonance the stability of the stationary planar oscillation of the satellite show that the discontinuity occurs in the amplitude of the oscillation at a frequency of the external periodic force which is less than the frequency of the natural oscillation.

\section{REFERENCES}

1. Inarrea, M., Lanchares, V., 2000 Chaos in the reorientation process of a dual-spin spacecraft with time-dependent moments of inertia: International Journal of Bifurcation and chaos. 10: 997-1018.

2. Abd., F.A., Salam, E.I., Sehnal, L., 2004. A second order analytical atmospheric drag theory based on the TD88 thermospheric density model: Celestial Mechanics and Dynamical Astronomy, 90: 361389.

3. Maciejewski, A.J., Przybylska, M., 2003 Non Integrability of the Problem of a Rigid Satellite in Gravitational and Magnetic Fields: Celestial Mechanics and Dynamical Astronomy. 87: 317351 .

4 Barkin, Y. V., Ferrandiz, J. M., 2004. Resonant Rotation of Two-layer Moon and Mercury: American Astronomical Society, DDA meeting 35.

5. Callegari, N., S. Ferraz-Mello and T.A. Michtchenko, 2006. Dynamics of two planets in the 3/2 mean-motion resonance: Application to the planetary system of the pulsar PSR B1257+12. Celestial Mech. Dynam. Astron., 94: 381-397.
6 Beaugé, C., N. Callegari, S. Ferraz-Mello and T.A. Michtchenko, 2005. Resonances and stability of extra-solar planetary systems: Dynamics of populations of planetary systems. Proc. IAU Colloquium 197. Ed. Z. Knezevic and A. Milani, Cambridge: Cambridge University Press, pp: 3-18.

7. Beaugé, C., T.A. Michtchenko and S. FerrazMello, 2006 Planetary migration and extrasolar planets in the 2/1 mean-motion resonance. Monthly Notices of the Royal Astronomical Society, 365: 1160-1170.

8. Massimiliano, G., 2005. The web of three-planet resonances in the outer Solar System. Icarus, 174: 273-284.

9 Guzzo, Massimiliano, 2006 The web of threeplanet resonances in the outer Solar System. Icarus, 181: 475-485.

10. Zhou, J.-L. and Y.-S. Sun, 2004. Criteria for the Occurrence of Apsidal and Nodal Secular Resonance in Planetary Systems: Stars as Suns. Activity, Evolution and Planets, Ed., A.K. Dupree and A.O. Benz. San Francisco, Astronomical Society of the Pacific (ASP), pp: 820.

11. Li-Yong, Z., Y.-S. Sun and J.-Q. Zheng, 2004. Apsidal corotation in mean motion resonance: The 55 Cancri system as an example. Monthly Notices of the Royal Astronomical Society, 350: 14951502 .

12. Yokoyama, T., M.R. Mana, C. do Nascimento, M.T. Santos, N.Jr. Callegari, 2005. On the dynamics of some resonances of Phobos in the future. Astronomy and Astrophysics, 429: 731-738. 